\title{
Robust hedging with proportional transaction costs
}

\section{Journal Article}

\section{Author(s):}

Dolinsky, Yan; Soner, Mete (1)

Publication date:

2014-04

Permanent link:

https://doi.org/10.3929/ethz-b-000075737

\section{Rights / license:}

In Copyright - Non-Commercial Use Permitted

\section{Originally published in:}

Finance and Stochastics 18(2), https://doi.org/10.1007/s00780-014-0227-x 


\title{
Robust hedging with proportional transaction costs
}

\author{
Yan Dolinsky • H. Mete Soner
}

Received: 4 February 2013 / Accepted: 20 September 2013 / Published online: 4 March 2014

(C) Springer-Verlag Berlin Heidelberg 2014

\begin{abstract}
A duality for robust hedging with proportional transaction costs of pathdependent European options is obtained in a discrete-time financial market with one risky asset. The investor's portfolio consists of a dynamically traded stock and a static position in vanilla options, which can be exercised at maturity. Trading of both options and stock is subject to proportional transaction costs. The main theorem is a duality between hedging and a Monge-Kantorovich-type optimization problem. In this dual transport problem, the optimization is over all probability measures that satisfy an approximate martingale condition related to consistent price systems, in addition to an approximate marginal constraint.
\end{abstract}

Keywords European options · Robust hedging · Transaction costs · Weak convergence $\cdot$ Consistent price systems · Optimal transport · Fundamental theorem of asset pricing $\cdot$ Superreplication

Mathematics Subject Classification (2010) $91 \mathrm{G} 10 \cdot 60 \mathrm{G} 42$

JEL Classification G11 $\cdot$ G13 · D52

\section{Introduction}

As is well known, superreplication in markets with transaction costs is quite costly $[16,19]$. Naturally, the same is even more true for the model-free case in which

Y. Dolinsky

Dept. of Statistics, Hebrew University, Mount Scopus 4416, Jerusalem 91905, Israel

e-mail: yan.dolinsky@mail.huji.ac.il

H.M. Soner $(\bowtie)$

Dept. of Mathematics, and Swiss Finance Institute, ETH Zurich, Ramistrasse 101, 8092 Zurich, Switzerland

e-mail: hmsoner@ethz.ch 
one does not place any probabilistic assumptions on the behavior of the risky asset. However, one may reduce the hedging cost by including liquid derivatives in the superreplicating portfolio. In particular, one may use all call options (written on the underlying asset) with a price that is known to the investor initially. This leads us to the semistatic hedging introduced in the classical paper of Hobson [13] in markets without transaction costs. So, following [13], we assume that all call options are traded assets and can be initially bought or sold for a known price. In addition to these static option positions, the stock is also traded dynamically. These trades, however, are subject to transaction costs. Each option has its own cost, and their general structure is outlined in the next section.

In this market, we consider the problem of robust hedging of a given pathdependent European option. Robust hedging refers to superreplication of an option for all possible stock price processes. This approach has been actively researched over the past decade since the seminal paper of Hobson [13]. In particular, the optimal portfolio is explicitly constructed for special cases of European options in continuous time: barrier options in [4, 6, 7], lookback options in [11-13], and volatility options in [8]. The main technique that is employed in these papers is the Skorokhod embedding. For more information, we refer the reader to the surveys of Hobson [14], Obłój [17], and the references therein.

Recently, an alternative approach has been developed, which uses the connection to optimal transport. Duality results in different types of generality or modelling have been proved in [2, 3, 9, 11] in frictionless markets. In particular, [9] studies continuous-time models, [11] provides a connection to stochastic optimal control and proposes a general solution methodology, [3] proves a general duality in discrete time, and [2] studies the question of a fundamental theorem of asset pricing in this context.

Although much has been established, the effect of frictions - in particular the impact of transaction costs-in this context is not fully studied. The classical probabilistic models with transaction costs, however, are well studied. In the classical model, a stock price model is assumed, hedging is done only through the stock, and no static position in the options is used. Then the dual is given as the supremum of "approximate" martingale measures that are equivalent to the market probability measure; see $[15,18]$ and the references therein. In this paper, we extend this result to the robust case. Namely, we prove that the superreplication price can be represented as the value of a martingale optimal transport problem. The dual control problem is to find the supremum of the expectation of the option over all approximate martingale measures that also satisfy an approximate marginal condition at maturity. This result is stated in Theorem 2.6 below, and the definition of an approximate martingale is given in Definition 2.5. Indeed, approximate martingales are very closely related to consistent price systems, which play a central role in the duality theory for markets with proportional transaction costs.

Recently, Acciaio et al. [2] proved a fundamental theorem of asset pricing (FTAP) in discrete-time markets without transaction costs in the robust setting. Also, Bouchard and Nutz [5] study the FTAP again in discrete time, but in the quasi-sure setting. Our main duality result has implications toward these results as well. These corollaries are discussed in Sect. 2.5. 
As in our previous paper [9] on robust hedging, our proof relies on a discretization of the problem. We first show that the original robust hedging problem can be obtained as a limit of hedging problems that are defined on finite spaces. We exploit the finiteness of these approximate problems and directly apply an elementary KuhnTucker duality theory. We then prove that any sequence of probability measures that are asymptotically maximizers of these finite problems is tight. The final step is then to directly use weak convergence and pass to the limit.

The paper is organized as follows. The main results are formulated in the next section and proved in Sect. 3. The final section is devoted to the proof of an auxiliary result that is used in the proof of the main results. This auxiliary result deals with superreplication under constraints and may be of independent interest.

\section{Preliminaries and main results}

The financial market consists of a savings account $B$ and a risky asset $S$, and trading is restricted to finitely many time points. Hence, the stock price process is $S_{k}$, $k=0,1, \ldots, N$, where $N<\infty$ is the maturity date or total number of allowed trades. By discounting we normalize to $B \equiv 1$. Furthermore, we normalize the initial stock price $s:=S_{0}>0$ to 1 as well. Then the set $\Omega$ of all possible price processes is simply the set of all vectors $\left(\omega_{0}, \ldots, \omega_{N}\right) \in \mathbb{R}_{+}^{N+1}$ that satisfy $\omega_{0}=1$ and $\omega_{1}, \ldots, \omega_{N} \geq 0$. We let $\mathbb{S}$ be the canonical process given by $\mathbb{S}_{k}(\omega):=\omega_{k}$ for $k=0, \ldots, N$. We write $\omega$ or $\mathbb{S}$ for the elements of $\Omega$, depending on the context; $\omega$ is used for a function on the space, and $\mathbb{S}$ for the stock price process. Let us emphasize that we make no other assumptions on our financial market. In particular, we do not assume any probabilistic structure.

\subsection{An assumption on the European claim}

We consider general path-dependent options. Hence, the payoff is $X=G(\mathbb{S})$ with any function $G: \Omega \rightarrow \mathbb{R}$. Our approach requires us to make the following regularity and growth assumption. Let $\|\omega\|:=\max _{0 \leq k \leq n}\left|\omega_{k}\right|$ for $\omega \in \Omega$. We impose the following:

Assumption 2.1 $G$ is upper semi-continuous and bounded by a quadratic function, i.e., there exists a constant $L>0$ such that

$$
|G(\omega)| \leq L\left(1+\|\omega\|^{2}\right) \quad \forall \omega \in \Omega .
$$

The above assumption is quite general and allows most of the standard claims such as Asian, lookback, volatility, and barrier options. We use the quadratic growth for specificity and also to include volatility options. However, any growth condition with any exponent is sufficient for our results. In this paper, we choose not to include such an extension to avoid more technicalities. 


\subsection{Semi-static hedging with transaction costs}

Let $\kappa \geq 0$ be a given constant. Consider a model in which every purchase or sale of the risky asset at any time is subject to a proportional transaction cost of rate $\kappa$. We assume that $\kappa<1 / 4$. Then a portfolio strategy is a pair $\pi:=(f, \gamma)$, where $f: \mathbb{R}_{+} \rightarrow \mathbb{R}$ is a continuous function, and

$$
\gamma:\{0,1, \ldots, N-1\} \times \Omega \rightarrow \mathbb{R}
$$

is a progressively measurable map, i.e., $\gamma(i, \omega)=\gamma(i, \tilde{\omega})$ if $\omega_{j}=\tilde{\omega}_{j}$ for all $j \leq i$. The function $f$ represents the European option with payoff $f\left(\mathbb{S}_{N}\right)$ that is bought at time zero for the price of $\mathcal{P}(f)$, and $\gamma(k, \mathbb{S})$ represents the number of stocks that the investor invests at time $k$ given that the stock prices up to time $k$ are $\mathbb{S}_{0}, \mathbb{S}_{1}, \ldots, \mathbb{S}_{k}$. Then the portfolio value at the maturity date is given by

$$
Y_{N}^{\pi}(\mathbb{S}):=f\left(\mathbb{S}_{N}\right)+\sum_{i=0}^{N-1} \gamma(i, \mathbb{S})\left(\mathbb{S}_{i+1}-\mathbb{S}_{i}\right)-\kappa \sum_{i=0}^{N-1} \mathbb{S}_{i}|\gamma(i, \mathbb{S})-\gamma(i-1, \mathbb{S})|,
$$

where we set $\gamma(-1, \cdot) \equiv 0$. The initial cost of any portfolio $(f, \gamma)$ is the price of the option $\mathcal{P}(f)$. Properties of this price operator $\mathcal{P}$ are given in the next subsection.

Definition 2.2 A portfolio $\pi$ is called perfect (or perfectly dominating) if it superreplicates the option, i.e.,

$$
Y_{N}^{\pi}(\mathbb{S}) \geq G(\mathbb{S}) \quad \forall \mathbb{S} \in \Omega
$$

The minimal superreplication cost is given by

$$
V(G)=\inf \{\mathcal{P}(f) \mid \pi:=(f, \gamma) \text { is a perfect portfolio }\} .
$$

\subsection{European options and their prices}

We postulate a general pricing operator $\mathcal{P}(f)$ for the initial price of the option $f\left(\mathbb{S}_{N}\right)$. We assume that it has the following properties.

Assumption 2.3 There exists $p>2$ such that for the power function $x \mapsto x^{p}$, we have $\mathcal{P}\left(x^{p}\right)<\infty$. Consider the vector space

$$
\mathcal{H}:=\left\{f: \mathbb{R}_{+} \rightarrow \mathbb{R} \text { continuous } \mid \exists C>0 \text { such that }|f(x)| \leq C\left(1+x^{p}\right) \forall x \in \mathbb{R}_{+}\right\} .
$$

We assume that $\mathcal{P}: \mathcal{H} \rightarrow \mathbb{R}$ is a convex function and that for every constant $a \in \mathbb{R}$

$$
\mathcal{P}(a)=a .
$$

We also assume that $\mathcal{P}$ is positively homogeneous of degree one, i.e.,

$$
\mathcal{P}(\lambda f)=\lambda \mathcal{P}(f), \quad f \in \mathcal{H}, \lambda>0 .
$$


Furthermore, for every sequence $\left\{f_{n}\right\}_{n=1}^{\infty} \subset \mathcal{H}$ converging pointwise to $f \in \mathcal{H}$,

$$
\mathcal{P}(f) \geq \limsup _{n \rightarrow \infty} \mathcal{P}\left(f_{n}\right) .
$$

In (2.2) we assume that the function $f$ belongs to $\mathcal{H}$. We set $\mathcal{P}(f)=\infty$ for any $f \notin \mathcal{H}$. Hence, in (2.2) we consider only functions that belong to $\mathcal{H}$.

We conclude this section with an elementary result.

Lemma 2.4 The minimal superreplication cost $V$ is subadditive and positively homogeneous of degree one, i.e.,

$$
V(\lambda G)=\lambda V(G), \quad \lambda>0,
$$

and

$$
V(G+H) \leq V(G)+V(H) .
$$

Furthermore, if $G \geq 0$ and $V(G)<0$, then $V(G)=-\infty$.

Proof From the convexity and positive homogeneity of $\mathcal{P}$, it follows that $\mathcal{P}$ is subadditive, i.e., $\mathcal{P}(f+g) \leq \mathcal{P}(f)+\mathcal{P}(g)$. Thus, the first two properties follow immediately from (2.2). Finally, let $G \geq 0$ be a nonnegative claim and assume that $V(G)<0$. Then there exists a perfect portfolio $(f, \gamma)$ with $\mathcal{P}(f)<0$. Clearly, for any $\lambda>1,(\lambda f, \lambda \gamma)$ is also a perfect portfolio. Thus, from (2.3) we get $V(G) \leq \lim _{\lambda \rightarrow \infty} \mathcal{P}(\lambda f)=-\infty$, as claimed.

\subsection{The main result}

To state the main result of the paper, we need to introduce some probabilistic structure as well. Recall the space $\Omega$ and the canonical process $\mathbb{S}$. Let $\mathbb{F}=\left(\mathcal{F}_{k}\right)_{k=0}^{N}$ be the canonical filtration generated by the process $\mathbb{S}$, i.e., $\mathcal{F}_{k}=\sigma\left(\mathbb{S}_{0}, \ldots, \mathbb{S}_{k}\right)$.

Definition 2.5 A probability measure $\mathbb{Q}$ on $\left(\Omega, \mathcal{F}_{N}\right)$ is called a $\kappa$-approximate martingale law if $\mathbb{S}_{0}=1 \mathbb{Q}$-a.s. and if the pair $(\mathbb{Q}, \tilde{\mathbb{S}})$ with

$$
\tilde{\mathbb{S}}_{k}:=\mathbb{E}_{\mathbb{Q}}\left[\mathbb{S}_{N} \mid \mathcal{F}_{k}\right], \quad k=1, \ldots, N
$$

is a consistent price system in the sense of $[15,18]$, i.e., for any $k<N$,

$$
(1-\kappa) \mathbb{S}_{k} \leq \tilde{\mathbb{S}}_{k} \leq(1+\kappa) \mathbb{S}_{k} \quad \mathbb{Q} \text {-a.s. }
$$

We denote by $\mathcal{M}_{\kappa, \mathcal{P}}$ the set of all $\kappa$-approximate martingale laws $\mathbb{Q}$ such that

$$
\mathbb{E}_{\mathbb{Q}}\left[f\left(\mathbb{S}_{N}\right)\right] \leq \mathcal{P}(f) \quad \forall f \in \mathcal{H}
$$

The following theorem is the main result of the paper. We use the standard convention that the supremum over an empty set is equal to minus infinity. 
Theorem 2.6 Suppose that $G$ satisfies Assumption 2.1 and $\mathcal{P}$ satisfies Assumption 2.3. Then

$$
V(G)=\sup _{\mathbb{Q} \in \mathcal{M}_{\kappa, \mathcal{P}}} \mathbb{E}_{\mathbb{Q}}[G(\mathbb{S})]
$$

In particular, when the set of measures $\mathcal{M}_{\kappa, \mathcal{P}}$ is empty, $V(G)=-\infty$ for every $G$ satisfying Assumption 2.1.

Proof In view of (2.1) and the convention $\gamma(-1, \cdot) \equiv 0$, for any portfolio $\pi=(f, \gamma)$,

$$
\begin{aligned}
Y_{N}^{\pi}(\mathbb{S})= & f\left(\mathbb{S}_{N}\right)+\sum_{i=0}^{N-1} \sum_{j=0}^{i}\left(\gamma(j, \mathbb{S})-\gamma\left(j-1, \mathbb{S}_{)}\right)\left(\mathbb{S}_{i+1}-\mathbb{S}_{i}\right)\right. \\
& -\kappa \sum_{i=0}^{N-1} \mathbb{S}_{i}|\gamma(i, \mathbb{S})-\gamma(i-1, \mathbb{S})| \\
= & f\left(\mathbb{S}_{N}\right)+\sum_{j=0}^{N-1}(\gamma(j, \mathbb{S})-\gamma(j-1, \mathbb{S}))\left(\sum_{i=j}^{N-1}\left(\mathbb{S}_{i+1}-\mathbb{S}_{i}\right)\right) \\
& -\kappa \sum_{i=0}^{N-1} \mathbb{S}_{i}|\gamma(i, \mathbb{S})-\gamma(i-1, \mathbb{S})| \\
= & f\left(\mathbb{S}_{N}\right)+\sum_{j=0}^{N-1}(\gamma(j, \mathbb{S})-\gamma(j-1, \mathbb{S}))\left(\mathbb{S}_{N}-\mathbb{S}_{j}\right) \\
& -\kappa \sum_{i=0}^{N-1} \mathbb{S}_{i}|\gamma(i, \mathbb{S})-\gamma(i-1, \mathbb{S})| .
\end{aligned}
$$

Suppose that $\mathcal{M}_{\kappa, \mathcal{P}}$ is nonempty. Let $\mathbb{Q} \in \mathcal{M}_{\kappa, \mathcal{P}}$, and let $\pi=(f, \gamma)$ be a perfect portfolio. Then (2.5) and (2.6) yield that

$$
\begin{aligned}
& \mathbb{E}_{\mathbb{Q}}[G(\mathbb{S})] \leq \mathbb{E}_{\mathbb{Q}}\left[Y_{N}^{\pi}(\mathbb{S})\right] \\
& \leq \mathcal{P}(f)+\sum_{i=0}^{N-1} \mathbb{E}_{\mathbb{Q}}\left[(\gamma(i, \mathbb{S})-\gamma(i-1, \mathbb{S}))\left(\tilde{\mathbb{S}}_{i}-\mathbb{S}_{i}\right)\right] \\
&-\kappa \sum_{i=0}^{N-1} \mathbb{E}_{\mathbb{Q}}\left[\mathbb{S}_{i}|\gamma(i, \mathbb{S})-\gamma(i-1, \mathbb{S})|\right] \\
& \leq \mathcal{P}(f) .
\end{aligned}
$$

So we have proved that

$$
\sup _{\mathbb{Q} \in \mathcal{M}_{\kappa, \mathcal{P}}} \mathbb{E}_{\mathbb{Q}}[G(\mathbb{S})] \leq V(G)
$$


Hence, to complete the proof of the theorem, it suffices to show that

$$
V(G) \leq \sup _{\mathbb{Q} \in \mathcal{M}_{\kappa, \mathcal{P}}} \mathbb{E}_{\mathbb{Q}}[G(\mathbb{S})]
$$

The proof of (2.8) is given in the next section.

Remark 2.7 Consider the following more general problem. Assume that for $0<k \leq N$ and a set of times $0<i_{1}<i_{2}<\cdots<i_{k}=N$, one can initially buy vanilla options with a payoff $f_{j}\left(\mathbb{S}_{i_{j}}\right)$ with maturity date $i_{j}$ for the price $\mathcal{P}_{j}\left(f_{j}\right)$, where $\mathcal{P}_{1}, \ldots, \mathcal{P}_{k}$ satisfy assumptions similar to Assumption 2.3. Then, by using the same approach in a recursive manner, we may extend Theorem 2.6 to prove that the superreplication cost in this context is equal to

$$
\sup _{\mathbb{Q} \in \mathcal{M}_{\kappa}, \mathcal{P}_{1}, \ldots, \mathcal{P}_{k}} \mathbb{E}_{\mathbb{Q}}[G(\mathbb{S})]
$$

where $\mathcal{M}_{\kappa, \mathcal{P}_{1}, \ldots, \mathcal{P}_{k}}$ is the set of all $\kappa$-approximate probability laws $\mathbb{Q}$ such that for any time $j=1, \ldots, k$ and $f \in \mathcal{H}$, we have

$$
\mathbb{E}_{\mathbb{Q}}\left[f\left(\mathbb{S}_{i_{j}}\right)\right] \leq \mathcal{P}_{j}(f)
$$

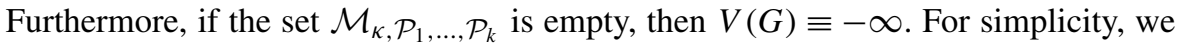
deal in this paper only with the case $k=1$.

\subsection{Fundamental theorem of asset pricing}

Theorem 2.6 also implies results that can be seen as a fundamental theorem of asset pricing (FTAP) for this market. Indeed, when the set $\mathcal{M}_{\kappa, \mathcal{P}}$ of measures is empty, we conclude by Theorem 2.6 that the minimal superreplication cost of any $G$ (satisfying Assumption 2.1) is equal to minus infinity. This is a clear indication of arbitrage. However, to make a precise statement, we need to define the notion of arbitrage. Since we do not assume a probabilistic structure, there are at least two possible approaches. Indeed, in frictionless markets, an FTAP is proved under different assumptions and definitions in [2] and in [5]. Our result essentially implies an FTAP under both definitions under Assumption 2.3.

Definition 2.8 We say that the model admits

- no model-independent arbitrage $\left(\mathrm{NA}_{\mathrm{mi}}\right)$ if for every $G \geq 0$ satisfying Assumption 2.1, we have $V(G) \geq 0$.

- no local arbitrage $\left(\mathrm{NA}_{\text {local }}\right)$ if for every continuous, bounded $G \geq 0, G \not \equiv 0$, we have $V(G)>0$.

In the above definition, $\mathrm{NA}_{\mathrm{mi}}$ is similar to the notion used in [2]. Also, a closely related definition is given by Cox and Obłój [6]. On the other hand, $\mathrm{NA}_{\text {local }}$ is analogous to the one used in [5]. One may also consider other versions of $\mathrm{NA}_{\text {local }}$ by requiring different notions of regularity of $G$. In the probabilistic setting, this is related to the 
choice of the polar sets. There one requires the set $\{G>0\}$ to be nonpolar (cf. [5]). Clearly, other possible regularity choices in $\mathrm{NA}_{\text {local }}$ would yield different equivalent conditions than the one proved in the second part of the following corollary. They can be proved similarly. We do not elaborate on different choices.

Corollary 2.9 Suppose that $\mathcal{P}$ satisfies Assumption 2.3.

1. There is no model-independent arbitrage if and only if $\mathcal{M}_{\kappa, \mathcal{P}}$ is nonempty. In particular, $N A_{\mathrm{mi}}$ holds if and only if there is at least one $G$ satisfying Assumption 2.1 with $V(G)>-\infty$.

2. There is no local arbitrage if and only if for every open subset $O \subset \Omega$, there is $\mathbb{Q} \in \mathcal{M}_{\kappa, \mathcal{P}}$ with $\mathbb{Q}[O]>0$.

Proof The first statement follows immediately from Theorem 2.6. So we only prove the second one. First, assume that $\mathrm{NA}_{\text {local }}$ holds. Let $O \subset \Omega$ be an arbitrary open set. Set

$$
G_{O}(\omega):=\min \{1, \operatorname{dist}(\omega, \Omega \backslash O)\} .
$$

Since $G_{O}$ is bounded and continuous, by $\mathrm{NA}_{\text {local }}, V\left(G_{O}\right)>0$. Since $0 \leq G_{O} \leq 1$ and $G_{O}=0$ outside of $O$, by Theorem 2.6,

$$
0<V\left(G_{O}\right)=\sup _{\mathbb{Q} \in \mathcal{M}_{\kappa, \mathcal{P}}} \mathbb{E}_{\mathbb{Q}}\left[G_{O}(\mathbb{S})\right] \leq \sup _{\mathbb{Q} \in \mathcal{M}_{\kappa, \mathcal{P}}} \mathbb{Q}[O]
$$

Hence, there must exist a measure $\mathbb{Q} \in \mathcal{M}_{\kappa, \mathcal{P}}$ with $\mathbb{Q}[O]>0$.

To prove the opposite implication, consider a continuous, bounded option $G \geq 0$, $G \not \equiv 0$. Set

$$
O_{G}:=\{\omega \in \Omega: G(\omega)>0\} .
$$

By the continuity of $G, O_{G}$ is a nonempty, open set. By hypothesis, there exists $\mathbb{Q}_{G} \in \mathcal{M}_{\kappa, \mathcal{P}}$ with $\mathbb{Q}_{G}\left[O_{G}\right]>0$. We estimate using Theorem 2.6 to arrive at

$$
V(G)=\sup _{\mathbb{Q} \in \mathcal{M}_{\kappa, \mathcal{P}}} \mathbb{E}_{\mathbb{Q}}[G(\mathbb{S})] \geq \mathbb{E}_{\mathbb{Q}_{G}}[G(\mathbb{S})]>0
$$

\section{Proof of the main result}

In this section, we prove (2.8).

3.1 Reduction to bounded uniformly continuous claims

We first use the elegant pathwise approach of [1] to martingale inequalities to show that the superreplication costs of certain options are asymptotically small. Indeed, for $M>0$, consider the option

$$
\alpha_{M}(\mathbb{S}):=\|\mathbb{S}\|^{2} \chi\{\|\mathbb{S}\| \geq M\}
$$


Let $\mathbb{S}^{*}$ be the running maximum, i.e.,

$$
\mathbb{S}_{k}^{*}:=\max _{0 \leq i \leq k} \mathbb{S}_{i}
$$

Since $\mathbb{S}_{k} \geq 0$ for each $k,\|\mathbb{S}\|=\mathbb{S}_{N}^{*}$.

\section{Lemma 3.1}

$$
\lim _{M \rightarrow \infty} \sup _{\mathbb{Q} \in \mathcal{M}_{\kappa, \mathcal{P}}} \mathbb{E}_{\mathbb{Q}}\left[\alpha_{M}(\mathbb{S})\right] \leq \lim _{M \rightarrow \infty} V\left(\alpha_{M}\right) \leq 0
$$

Proof Let $p>2$ be the exponent in Assumption 2.3. Since $\kappa<1 / 4$, there exists $r \in(2, p)$ such that $\lambda:=\kappa r c_{r}<1$, with

$$
c_{r}:=\frac{r}{r-1} .
$$

We now use Proposition 2.1 in [1] with the portfolio $\hat{\pi}=(\hat{f}, \hat{\gamma})$ given by

$$
\hat{f}\left(\mathbb{S}_{N}\right):=\left(c_{r} \mathbb{S}_{N}\right)^{r}-c_{r}, \quad \hat{\gamma}(i, \mathbb{S})=-r c_{r}\left(\mathbb{S}_{k}^{*}\right)^{r-1}, \quad k<N .
$$

We use (2.1) and Proposition 2.1 in [1] to arrive at

$$
\begin{aligned}
Y_{N}^{\hat{\pi}}(\mathbb{S}) & \geq\|\mathbb{S}\|^{r}-\kappa \sum_{i=0}^{N-1} \mathbb{S}_{i}|\hat{\gamma}(i, \mathbb{S})-\hat{\gamma}(i-1, \mathbb{S})| \\
& \geq\|\mathbb{S}\|^{r}-\kappa\|\mathbb{S}\| \sum_{i=0}^{N-1}(\hat{\gamma}(i-1, \mathbb{S})-\hat{\gamma}(i, \mathbb{S}))=\|\mathbb{S}\|^{r}(1-\lambda) .
\end{aligned}
$$

Hence,

$$
V\left((1-\lambda)\|\mathbb{S}\|^{r}\right) \leq \mathcal{P}(\hat{f}) .
$$

Clearly, $\alpha_{M}(\mathbb{S}) \leq\|\mathbb{S}\|^{r} / M^{r-2}$. Hence, by Lemma 2.4,

$$
V\left(\alpha_{M}\right) \leq V\left(\frac{\|\mathbb{S}\|^{r}}{M^{r-2}}\right)=\frac{1}{(1-\lambda) M^{r-2}} V\left((1-\lambda)\|\mathbb{S}\|^{r}\right) \leq \frac{1}{(1-\lambda) M^{r-2}} \mathcal{P}(\hat{f}) .
$$

Since $\hat{f} \in \mathcal{H}, \mathcal{P}(\hat{f})$ is finite. Therefore,

$$
\lim _{M \rightarrow \infty} V\left(\alpha_{M}\right) \leq 0 .
$$

To complete the proof, we recall the proof of (2.7) to restate that for every $M$,

$$
\sup _{\mathbb{Q} \in \mathcal{M}_{\kappa, \mathcal{P}}} \mathbb{E}_{\mathbb{Q}}\left[\alpha_{M}(\mathbb{S})\right] \leq V\left(\alpha_{M}\right)
$$

The preceding result allows us to consider bounded claims. We also use a compactness argument to obtain the following equivalence. 
Theorem 3.2 It suffices to prove (2.8) for nonnegative bounded uniformly continuous claims.

Since the proof of this result is almost orthogonal to the rest of the paper, we relegate it to the Appendix.

In view of Theorem 3.2, we assume in the sequel that the claim $G$ is nonnegative, bounded, and uniformly continuous. So we assume that there exist a constant $K>0$ and a modulus of continuity, i.e., a continuous function $m: \mathbb{R}_{+} \rightarrow \mathbb{R}_{+}$with $m(0)=0$, satisfying

$$
0 \leq G(\omega) \leq K, \quad|G(\omega)-G(\tilde{\omega})| \leq m(\|\omega-\tilde{\omega}\|) \quad \forall \omega, \tilde{\omega} \in \Omega .
$$

If $V(G)=-\infty$, then (2.8) is clear. Thus, in view of Lemma 2.4, we can assume without loss of generality that

$$
V(G) \geq 0
$$

\subsection{Discretization of the space}

Next, we introduce a modification of the original superreplication problem. Fix $n \in \mathbb{N}$ and set $h=1 / n$ and $U_{n}=\{k h, k=0,1, \ldots\}$. Denote

$$
\mathcal{H}_{n}:=\left\{f: U_{n} \rightarrow \mathbb{R} \mid \exists C>0 \text { such that }|f(x)| \leq C\left(1+x^{p}\right) \forall x\right\} .
$$

For any $g: U_{n} \rightarrow \mathbb{R}$, define the function $\mathcal{L}^{(n)}(g): \mathbb{R}_{+} \rightarrow \mathbb{R}$ by

$$
\mathcal{L}^{(n)}(g)(x):=(1-\alpha) g(\lfloor n x\rfloor h)+\alpha g((\lfloor n x\rfloor+1) h), \quad \alpha=n x-\lfloor n x\rfloor,
$$

where for a real number $y,\lfloor y\rfloor$ is the largest integer less than or equal to $y$. Observe that

$$
\mathcal{L}^{(n)}: \mathcal{H}_{n} \rightarrow \mathcal{H}
$$

is a bounded linear map.

Set $\Omega_{n}=\left(U_{n}\right)^{N}$. Clearly, $\Omega_{n} \subset \Omega$, and we consider a financial market where the set of possible stock price processes is the set $\Omega_{n}$. Then this restriction lowers the minimal superreplication cost. However, we restrict the admissible portfolios as well. Indeed, for a constant $M>0$, we define the set of admissible portfolio strategies as follows.

Definition 3.3 For any $M>1$, we say that $\pi:=(g, \gamma)$ is an $(M$-)admissible portfolio if $g \in \mathcal{H}_{n}$ and $\gamma:\{0,1, \ldots, N-1\} \times \Omega_{n} \rightarrow \mathbb{R}$ is a progressively measurable map satisfying

$$
|\gamma(i, \mathbb{S})-\gamma(i-1, \mathbb{S})| \leq M \quad \forall i=1, \ldots, N, \mathbb{S} \in \Omega_{n} .
$$

We denote by $\mathcal{A}_{M}^{n}$ the set of all admissible portfolios. A portfolio $\pi \in \mathcal{A}_{M}^{n}$ is called perfect (or perfectly superreplicating) if

$$
Y_{N}^{\pi}(\mathbb{S}) \geq G(\mathbb{S}) \quad \forall \mathbb{S} \in \Omega_{n},
$$

where $Y_{N}^{\pi}$ is given by (2.1). 
The minimal superreplication cost is given by

$$
V^{n, M}(G):=\inf \left\{\mathcal{P}^{(n)}(g) \mid \pi:=(g, \gamma) \in \mathcal{A}_{M}^{n} \text { is a perfect portfolio }\right\},
$$

where we choose the price function as

$$
\mathcal{P}^{(n)}(g):=\mathcal{P}\left(\mathcal{L}^{(n)}(g)\right) .
$$

The following result provides the crucial connection between the original and the discretized problems. Recall that $h=1 / n$.

Proposition 3.4 Assume that $G$ satisfies (3.1) with a modulus function $m$. Then, for any $M>0$ and $n \in \mathbb{N}$,

$$
V(G) \leq V^{n, M}(G)+(N+2 \kappa) M N h+m(h) .
$$

Proof Assume that we have a perfect hedge $\pi=(g, \gamma) \in \mathcal{A}_{M}^{n}$ in the sense of Definition 3.3. We continue by lifting this portfolio to a portfolio $\tilde{\pi}=(f, \tilde{\gamma})$ that is defined on $\Omega$.

Let $f=\mathcal{L}^{(n)}(g)$ be as in (3.3) and define $\tilde{\gamma}$ by

$$
\tilde{\gamma}(k, \omega):=\gamma\left(k, \omega_{0},\left\lfloor n \omega_{1}\right\rfloor h, \ldots,\left\lfloor n \omega_{N}\right\rfloor h\right) \quad \forall k<N, \omega=\left(\omega_{0}, \ldots, \omega_{N}\right) \in \Omega,
$$

where as before $h=1 / n$. Clearly, $\tilde{\gamma}:\{0,1, \ldots, N-1\} \times \Omega \rightarrow \mathbb{R}$ is progressively measurable, and $|\tilde{\gamma}(i, \mathbb{S})-\tilde{\gamma}(i-1, \mathbb{S})| \leq M$ for any $i=1, \ldots, N$ and $\mathbb{S} \in \Omega$.

For $\mathbb{S} \in \Omega$, define $\mathbb{S}^{(1)}, \mathbb{S}^{(2)}$ by

$$
\mathbb{S}_{k}^{(1)}:=\left\lfloor n \mathbb{S}_{k}\right\rfloor h, \quad \mathbb{S}_{k}^{(2)}:=\mathbb{S}_{k}^{(1)}+h \delta_{k N} \quad \text { for all } k \leq N,
$$

where $\delta_{k N}$ is equal to one when $k=N$ and zero otherwise. Then there exists $\lambda \in[0,1]$ such that $\mathbb{S}_{N}=\lambda \mathbb{S}_{N}^{(1)}+(1-\lambda) \mathbb{S}_{N}^{(2)}$. Also, both $\left\|\mathbb{S}^{(1)}-\mathbb{S}\right\|$ and $\left\|\mathbb{S}^{(2)}-\mathbb{S}\right\|$ are less than $h=1 / n$. Moreover, $\gamma\left(k, \mathbb{S}^{(1)}\right)=\gamma\left(k, \mathbb{S}^{(2)}\right)=\tilde{\gamma}(k, \mathbb{S})$ for every $k<N$. We use these together with (2.1), (3.3), and the fact that $\gamma \in[-M N, M N]$. The result is

$$
\begin{aligned}
Y_{N}^{\tilde{\pi}}(\mathbb{S}) & \geq \lambda Y_{N}^{\pi}\left(\mathbb{S}^{(1)}\right)+(1-\lambda) Y_{N}^{\pi}\left(\mathbb{S}^{(2)}\right)-(N+2 \kappa) M N h \\
& \geq \lambda G\left(\mathbb{S}^{(1)}\right)+(1-\lambda) G\left(\mathbb{S}^{(2)}\right)-(N+2 \kappa) M N h \\
& \geq G(\mathbb{S})-m(h)-(N+2 \kappa) M N h,
\end{aligned}
$$

where the last inequality follows from (3.1). Thus, $(f+m(h)+(N+2 \kappa) M N h, \tilde{\gamma})$ is a perfect portfolio in the sense of Definition 2.2. This, together with the equality $\mathcal{P}_{n}(g)=\mathcal{P}(f)$, completes the proof.

\subsection{Analysis of $V^{n, \sqrt{n}}(G)$}

Fix $n>0$. From Proposition 3.4 and from (3.2) it follows that for sufficiently large $n$,

$$
V^{n, \sqrt{n}}(G) \geq-1 .
$$


Fix $n \in \mathbb{N}$ sufficiently large such that (3.6) holds. We introduce three auxiliary sets. Let $\mathcal{W}_{n}$ be the set of all functions $g \in \mathcal{H}_{n}$ that satisfy the growth condition

$$
\|g\|_{*}:=\sup _{x \in U_{n}} \frac{|g(x)|}{(1+x)^{p}} \leq n .
$$

Let $\mathcal{Q}_{n}$ be the set of all probability measures $\mathbb{Q}$ on $\Omega_{n}$ that satisfy

$$
\mathbb{E}_{\mathbb{Q}}\left[\|\mathbb{S}\|^{p}\right]<\infty
$$

Finally, let $\hat{\mathcal{Q}}_{n}$ be the set of all probability measures $\mathbb{Q} \in \mathcal{Q}_{n}$ that satisfy

$$
\mathbb{E}_{\mathbb{Q}}\left[g\left(\mathbb{S}_{N}\right)\right] \leq \mathcal{P}^{(n)}(g)+\frac{K+1}{n}\|g\|_{*} \quad \forall g \in \mathcal{W}_{n},
$$

where

$$
K:=\sup _{\mathbb{S} \in \Omega} G(\mathbb{S})
$$

We show in the proof below that in view of (3.6), the set of measures $\hat{\mathcal{Q}}_{n}$ is nonempty for all sufficiently large $n$.

The following provides an upper bound for the superreplication cost $V^{n, \sqrt{n}}$ defined by (3.4).

Lemma 3.5 Suppose that $G$ satisfies (3.1) and (3.2). Then, for all sufficiently large $n$,

$$
V^{n, \sqrt{n}}(G) \leq \sup _{\mathbb{Q} \in \hat{\mathcal{Q}}_{n}} \mathbb{E}_{\mathbb{Q}}\left[G(\mathbb{S})-\sqrt{n} \sum_{k=0}^{N-1}\left(\left|\mathbb{E}_{\mathbb{Q}}\left[\mathbb{S}_{N} \mid \mathcal{F}_{k}\right]-\mathbb{S}_{k}\right|-\kappa \mathbb{S}_{k}\right)^{+}\right]
$$

Proof Define $H: \mathcal{W}_{n} \times \mathcal{Q}_{n} \rightarrow \mathbb{R}$ by

$$
H(g, \mathbb{Q}):=\mathbb{E}_{\mathbb{Q}}\left[G(\mathbb{S})-g\left(\mathbb{S}_{N}\right)-\sqrt{n} \sum_{k=0}^{N-1}\left(\left|\mathbb{E}_{\mathbb{Q}}\left[\mathbb{S}_{N} \mid \mathcal{F}_{k}\right]-\mathbb{S}_{k}\right|-\kappa \mathbb{S}_{k}\right)^{+}\right]+\mathcal{P}^{(n)}(g)
$$

Since $\mathcal{P}^{(n)}$ is finite on $\mathcal{W}_{n}$, in view of the definitions of $\mathcal{W}_{n}$ and $\mathcal{Q}_{n}, H$ is well defined. We now use Theorem 4.1 (which will be stated and proved in the next section) with $F(\mathbb{S}):=G(\mathbb{S})-g\left(\mathbb{S}_{N}\right)$ for an arbitrary $g \in \mathcal{W}_{n}$. This yields that

$$
V^{n, \sqrt{n}}(G) \leq \sup _{\mathbb{Q} \in \mathcal{Q}_{n}} H(g, \mathbb{Q}) \quad \forall g \in \mathcal{W}_{n}
$$

Hence,

$$
V^{n, \sqrt{n}}(G) \leq \inf _{g \in W_{n}} \sup _{\mathbb{Q} \in \mathcal{Q}_{n}} H(g, \mathbb{Q}) .
$$

Since the functions in $\mathcal{W}_{n}$ are restricted to satisfy the growth condition (3.7), the above is possibly an inequality and not an equality. 
Next, we continue by interchanging the order of the above infimum and supremum. For that purpose, consider the vector space $\mathbb{R}^{U_{n}}$ of all functions $g: U_{n} \rightarrow \mathbb{R}$ with the topology of pointwise convergence. This space is locally convex, and since $U_{n}$ is countable, $\mathcal{W}_{n} \subset \mathbb{R}^{U_{n}}$ is compact. (Indeed, the notion of convergence is pointwise. So for any sequence $f_{k} \in W_{n}$ and at each $x \in U_{n}$, one can find a convergent subsequence $f_{n_{k}}(x)$. Then a diagonal argument yields a subsequence $m_{k}$ such that $f_{m_{k}}(x)$ is convergent at each point $x$.) Also, the set $\mathcal{Q}_{n}$ can be naturally considered as a convex subspace of the vector space $\mathbb{R}^{\Omega_{n}}$. In order to apply a minimax theorem, we need to show the continuity and concavity of $H$. In view of Assumption 2.3, $H$ is convex in the first variable and therefore continuous due to the dominated convergence theorem. We also claim that $H$ is concave in the second variable. For this, it is sufficient to show that for any $k<N$, the functional $\mathbb{E}_{\mathbb{Q}}\left[\left(\left|\mathbb{E}_{\mathbb{Q}}\left[\mathbb{S}_{N} \mid \mathcal{F}_{k}\right]-\mathbb{S}_{k}\right|-\kappa \mathbb{S}_{k}\right)^{+}\right]$ is convex in $\mathbb{Q}$. Indeed, this follows from the representation

$$
\begin{aligned}
\mathbb{E}_{\mathbb{Q}} & {\left[\left(\left|\mathbb{E}_{\mathbb{Q}}\left[\mathbb{S}_{N} \mid \mathcal{F}_{k}\right]-\mathbb{S}_{k}\right|-\kappa \mathbb{S}_{k}\right)^{+}\right] } \\
= & \sum_{\left(z_{1}, \ldots, z_{k}\right) \in U_{n}^{k}}\left(\left|\sum_{z_{N} \in U_{n}} z_{N} \mathbb{Q}[A]-z_{k} \mathbb{Q}[B]\right|-\kappa z_{k} \mathbb{Q}[B]\right)^{+},
\end{aligned}
$$

where

$$
\begin{aligned}
& A=A\left(z_{1}, \ldots, z_{k}, z_{N}\right):=\left\{\mathbb{S}_{1}=z_{1}, \ldots, \mathbb{S}_{k}=z_{k}, \mathbb{S}_{N}=z_{N}\right\}, \\
& B=B\left(z_{1}, \ldots, z_{k}\right):=\left\{\mathbb{S}_{1}=z_{1}, \ldots, \mathbb{S}_{k}=z_{k}\right\} .
\end{aligned}
$$

We now apply Theorem 45.8 in [20] to the function $H$. The result is

$$
\inf _{g \in \mathcal{W}_{n}} \sup _{\mathbb{Q} \in \mathcal{Q}_{n}} H(g, \mathbb{Q})=\sup _{\mathbb{Q} \in \mathcal{Q}_{n}} \inf _{g \in \mathcal{W}_{n}} H(g, \mathbb{Q}) .
$$

We combine this with (3.9) (and (3.10)) to obtain

$$
V^{n, \sqrt{n}}(G) \leq \sup _{\mathbb{Q} \in \mathcal{Q}_{n}} \inf _{g \in \mathcal{W}_{n}} H(g, \mathbb{Q}) .
$$

Now suppose that $\mathbb{Q}$ is in $\mathcal{Q}_{n}$ but not in $\hat{\mathcal{Q}}_{n}$. Then there is $g^{*} \in \mathcal{W}_{n}$ such that

$$
\mathbb{E}_{\mathbb{Q}}\left[g^{*}\left(\mathbb{S}_{N}\right)\right]>\mathcal{P}^{(n)}\left(g^{*}\right)+\frac{K+1}{n}\left\|g^{*}\right\|_{*} .
$$

By the positive homogeneity of $\mathcal{P}$ we may assume that $\left\|g^{*}\right\|_{*}=n$. Then we get

$$
\mathbb{E}_{\mathbb{Q}}\left[g^{*}\left(\mathbb{S}_{N}\right)\right]>\mathcal{P}^{(n)}\left(g^{*}\right)+K+1
$$

and recall that $K=\sup _{\Omega} G$. The definition of $H$ yields that

$$
H\left(g^{*}, \mathbb{Q}\right) \leq \mathbb{E}_{\mathbb{Q}}[G(\mathbb{S})]-\mathbb{E}_{\mathbb{Q}}\left[g^{*}\left(\mathbb{S}_{N}\right)\right]+\mathcal{P}^{(n)}\left(g^{*}\right)<\mathbb{E}_{\mathbb{Q}}[G(\mathbb{S})]-K-1 \leq-1 .
$$

In view of (3.6), we conclude that there must exist measures in $\hat{\mathcal{Q}}_{n}$. Additionally, we may restrict the maximization in (3.11) over the probability measures $\mathbb{Q} \in \hat{\mathcal{Q}}_{n}$. We 
use this restricted version of (3.11) to arrive at

$$
V^{n, \sqrt{n}}(G) \leq \sup _{\mathbb{Q} \in \hat{\mathcal{Q}}_{n}} \inf _{g \in \mathcal{W}_{n}} H(g, \mathbb{Q}) \leq \sup _{\mathbb{Q} \in \hat{\mathcal{Q}}_{n}} H(0, \mathbb{Q}) .
$$

Since $\mathcal{P}(0)=0$, the above is exactly the statement of the lemma.

\subsection{Last step of the proof}

We combine Proposition 3.4 and Lemma 3.5 to conclude that

$$
V(G) \leq \liminf _{n \rightarrow \infty} \beta_{n}
$$

where

$$
\beta_{n}:=\sup _{\mathbb{Q} \in \hat{\mathcal{Q}}_{n}} \mathbb{E}_{\mathbb{Q}}\left[G(\mathbb{S})-\sqrt{n} \sum_{k=0}^{N-1}\left(\left|\mathbb{E}_{\mathbb{Q}}\left[\mathbb{S}_{N} \mid \mathcal{F}_{k}\right]-\mathbb{S}_{k}\right|-\kappa \mathbb{S}_{k}\right)^{+}\right]
$$

Thus, in order to complete the proof of inequality (2.8), it is sufficient to establish the following:

Lemma 3.6 Suppose that $G$ satisfies (3.1) and (3.2). Then,

$$
\liminf _{n \rightarrow \infty} \beta_{n} \leq \sup _{\mathbb{Q} \in \mathcal{M}_{\kappa, \mathcal{P}}} \mathbb{E}_{\mathbb{Q}}[G(\mathbb{S})]
$$

Proof From (3.2) and (3.12) it follows that $\beta_{n} \geq-1$ for sufficiently large $n$. Therefore, for all sufficiently large $n \in \mathbb{N}$, there exists $\mathbb{Q}_{n} \in \hat{\mathcal{Q}}_{n}$ such that

$$
\mathbb{E}^{(n)}\left[\sum_{k=0}^{N-1}\left(\left|\mathbb{E}^{(n)}\left[\mathbb{S}_{N} \mid \mathcal{F}_{k}\right]-\mathbb{S}_{k}\right|-\kappa \mathbb{S}_{k}\right)^{+}\right] \leq \frac{K+1}{\sqrt{n}}
$$

and

$$
\beta_{n} \leq \frac{1}{n}+\mathbb{E}^{(n)}\left[G(\mathbb{S})-\sqrt{n} \sum_{k=0}^{N-1}\left(\left|\mathbb{E}^{(n)}\left[\mathbb{S}_{N} \mid \mathcal{F}_{k}\right]-\mathbb{S}_{k}\right|-\kappa \mathbb{S}_{k}\right)^{+}\right]
$$

where $\mathbb{E}^{(n)}$ denotes the expectation with respect to $\mathbb{Q}_{n}$. From (3.5) and (3.8), we get

$$
\mathbb{E}^{(n)}\left[\mathbb{S}_{N}^{p}\right] \leq \mathcal{P}^{(n)}\left(x^{p}\right)+\frac{K+1}{n}\left\|x^{p}\right\|_{*} \leq \mathcal{P}\left(2+2 x^{p}\right)+K+1<\infty .
$$

Hence,

$$
\sup _{n \in \mathbb{N}} \mathbb{E}^{(n)}\left[\mathbb{S}_{N}^{p}\right]<\infty
$$


We claim that the probability measures $\mathbb{Q}_{n}, n \in \mathbb{N}$, are tight. Indeed, in view of the uniform moment estimate (3.15), tightness will follow from uniform integrability, which states that for any $A>0$,

$$
\lim _{A \rightarrow \infty} \sup _{n \in \mathbb{N}} \mathbb{E}^{(n)}\left[\mathbb{S}_{k} \chi_{\left\{\mathbb{S}_{k}>A\right\}}\right]=0 \quad \forall k=1, \ldots, N-1 .
$$

Since $\mathbb{S}_{k}$ is $\mathbb{Q}_{n}$-integrable, the above will follow from

$$
\lim _{M \rightarrow \infty} \lim _{A \rightarrow \infty} \sup _{n \geq M} \mathbb{E}^{(n)}\left[\mathbb{S}_{k} \chi\left\{\mathbb{S}_{k}>A\right\}\right]=0 \quad \forall k=1, \ldots, N-1 .
$$

So we continue by proving (3.16). We first fix positive integers $k<N, n$ and set

$$
X:=(1-\kappa) \mathbb{S}_{k}, \quad Y:=\mathbb{E}^{(n)}\left[\mathbb{S}_{N} \mid \mathcal{F}_{k}\right] .
$$

In view of (3.13), $\mathbb{E}^{(n)}\left[(X-Y)^{+}\right] \leq(K+1) / \sqrt{n}$. Therefore, by the Cauchy-Schwarz and Markov inequalities, we obtain that for any $A>0$,

$$
\begin{aligned}
\mathbb{E}^{(n)}\left[X \chi_{\{X>A\}}\right] & \leq \mathbb{E}^{(n)}\left[\left((X-Y)^{+}+Y\right) \chi_{\{X>A\}]}\right. \\
& \leq \mathbb{E}^{(n)}\left[(X-Y)^{+}\right]+\mathbb{E}^{(n)}\left[Y \chi_{\{X>A\}}\right] \\
& \leq \frac{K+1}{\sqrt{n}}+\mathbb{E}^{(n)}\left[Y \chi_{\{X>A\}}\right] \\
& \leq \frac{K+1}{\sqrt{n}}+\sqrt{\mathbb{E}^{(n)}\left[Y^{2}\right]} \sqrt{\mathbb{Q}_{n}[X>A]} \\
& \leq \frac{K+1}{\sqrt{n}}+\sqrt{\mathbb{E}^{(n)}\left[\mathbb{S}_{N}^{2}\right]} \sqrt{\mathbb{E}^{(n)}[X]} \frac{1}{\sqrt{A}} \\
& \leq \frac{K+1}{\sqrt{n}}+\frac{1}{\sqrt{A}} \sqrt{\mathbb{E}^{(n)}\left[\mathbb{S}_{N}^{2}\right]} \sqrt{\frac{K+1}{\sqrt{n}}+\mathbb{E}^{(n)}[Y]} \\
& \leq \frac{K+1}{\sqrt{n}}+\frac{1}{\sqrt{A}} \sqrt{\mathbb{E}^{(n)}\left[\mathbb{S}_{N}^{2}\right]} \sqrt{\frac{K+1}{\sqrt{n}}+\mathbb{E}^{(n)}\left[\mathbb{S}_{N}\right]}
\end{aligned}
$$

This together with (3.15) yields (3.16) and hence the uniform integrability of the sequence $\mathbb{Q}_{n}, n \in \mathbb{N}$.

In view of Prokhorov's theorem, there now exists a subsequence $\mathbb{Q}_{n_{\ell}}, \ell \in \mathbb{N}$, that converges weakly to a probability measure $\tilde{\mathbb{Q}}$. Then, (3.14) implies that

$$
\begin{aligned}
\tilde{\mathbb{E}}[G(\mathbb{S})] & =\lim _{\ell \rightarrow \infty} \mathbb{E}^{\left(n_{\ell}\right)}[G(\mathbb{S})] \\
& \geq \liminf _{n \rightarrow \infty} \sup _{\mathbb{Q} \in \hat{\mathcal{Q}}_{n}} \mathbb{E}_{\mathbb{Q}}\left[G(\mathbb{S})-\sqrt{n} \sum_{k=0}^{N-1}\left(\left|\mathbb{E}_{\mathbb{Q}}\left[\mathbb{S}_{N} \mid \mathcal{F}_{k}\right]-\mathbb{S}_{k}\right|-\kappa \mathbb{S}_{k}\right)^{+}\right],
\end{aligned}
$$

where $\tilde{\mathbb{E}}$ denotes the expectation with respect to $\tilde{\mathbb{Q}}$. Then, Proposition 3.4 and Lemma 3.5 imply (2.8), provided that $\tilde{\mathbb{Q}} \in \mathcal{M}_{\kappa, \mathcal{P}}$. Thus, in order to complete the 
proof of this lemma, it suffices to show that the limit probability measure $\tilde{\mathbb{Q}}$ is in $\mathcal{M}_{\kappa, \mathcal{P}}$.

Fix $k$ and let $h: \mathbb{R}^{k} \rightarrow \mathbb{R}_{+}$be a continuous bounded function. Denote by $\|\cdot\|$ the usual sup-norm on functions. By (3.13), it follows that

$$
\begin{aligned}
\mathbb{E}^{(n)}\left[\mathbb{S}_{N} h\left(\mathbb{S}_{1}, \ldots, \mathbb{S}_{k}\right)\right] & =\mathbb{E}^{(n)}\left[\mathbb{E}^{(n)}\left[\mathbb{S}_{N} \mid \mathcal{F}_{k}\right] h\left(\mathbb{S}_{1}, \ldots, \mathbb{S}_{k}\right)\right] \\
& \leq \mathbb{E}^{(n)}\left[(1+\kappa) \mathbb{S}_{k} h\left(\mathbb{S}_{1}, \ldots, \mathbb{S}_{k}\right)\right]+\frac{(K+1)\|h\|}{\sqrt{n}} .
\end{aligned}
$$

Similarly, we conclude that

$$
\begin{aligned}
\mathbb{E}^{(n)}\left[\mathbb{S}_{N} h\left(\mathbb{S}_{1}, \ldots, \mathbb{S}_{k}\right)\right] & =\mathbb{E}^{(n)}\left[\mathbb{E}^{(n)}\left[\mathbb{S}_{N} \mid \mathcal{F}_{k}\right] h\left(\mathbb{S}_{1}, \ldots, \mathbb{S}_{k}\right)\right] \\
& \geq \mathbb{E}^{(n)}\left[(1-\kappa) \mathbb{S}_{k} h\left(\mathbb{S}_{1}, \ldots, \mathbb{S}_{k}\right)\right]-\frac{(K+1)\|h\|}{\sqrt{n}} .
\end{aligned}
$$

We next take the limit $n_{\ell} \rightarrow \infty$ and use (3.15) and (3.16). The result is

$$
(1-\kappa) \tilde{\mathbb{E}}\left[\mathbb{S}_{k} h\left(\mathbb{S}_{1}, \ldots, \mathbb{S}_{k}\right)\right] \leq \tilde{\mathbb{E}}\left[\mathbb{S}_{N} h\left(\mathbb{S}_{1}, \ldots, \mathbb{S}_{k}\right)\right] \leq(1+\kappa) \tilde{\mathbb{E}}\left[\mathbb{S}_{k} h\left(\mathbb{S}_{1}, \ldots, \mathbb{S}_{k}\right)\right]
$$

The above holds for any nonnegative continuous bounded function $h$. Then by a standard density argument, we arrive at

$$
(1-\kappa) \mathbb{S}_{k} \leq \tilde{\mathbb{E}}\left[\mathbb{S}_{N} \mid \mathcal{F}_{k}\right] \leq(1+\kappa) \mathbb{S}_{k}, \quad k=0, \ldots, N-1 .
$$

Hence, $\tilde{\mathbb{Q}}$ is a $\kappa$-approximate martingale law.

We continue by showing that $\tilde{\mathbb{Q}}$ satisfies (2.6). From (3.15) it follows that

$$
\tilde{\mathbb{E}}\left[\mathbb{S}_{N}^{p}\right]<\infty .
$$

Let $g \in \mathcal{H}$, and let $C>0$ be such that $g(x) \leq C(1+x)^{p}$ for all $x \geq 0$. Set $f_{n}:=\left.g\right|_{U_{n}}$ and $h_{n}=\mathcal{L}_{n}\left(f_{n}\right), n \in \mathbb{N}$. Observe that for sufficiently large $n, f_{n} \in \mathcal{W}_{n}$. Since $g$ is continuous, $g(x)=\lim _{n \rightarrow \infty} f_{n}\left(x_{n}\right)$ for any $x \geq 0$ and any sequence $x_{n} \geq 0, n \in \mathbb{N}$, that converges to $x$. Furthermore, the sequence $h_{n}, n \in \mathbb{N}$, converges pointwise to $g$. We use the Skorokhod representation theorem, (2.4) and (3.5) to conclude that

$$
\tilde{\mathbb{E}}\left[g\left(\mathbb{S}_{N}\right)\right]=\lim _{n \rightarrow \infty} \mathbb{E}^{(n)}\left[f_{n}\left(\mathbb{S}_{N}\right)\right] \leq \liminf _{n \rightarrow \infty} \mathcal{P}^{(n)}\left(f_{n}\right)=\liminf _{n \rightarrow \infty} \mathcal{P}\left(h_{n}\right) \leq \mathcal{P}(g),
$$

as desired.

\section{Hedging with constraints and transaction costs}

This section is devoted to the proof of an auxiliary result that is used in Lemma 3.5.

Fix $n \in \mathbb{N}$ and recall that $\Omega_{n}=\{k h \mid k=0,1, \ldots\}^{N}$ with $h=1 / n$ as defined in Sect. 3.2. In this section, we do not allow one to buy vanilla options, but only to trade the stock with proportional transaction costs. Furthermore, the number of stocks that the investor is allowed to buy should lie in the interval $[-M, M]$. Therefore, in this 
section, a portfolio strategy is a pair $\tilde{\pi}=(x, \gamma)$, where $x \in \mathbb{R}$ is the initial capital, and $\gamma:\{0,1, \ldots, N-1\} \times \Omega_{n} \rightarrow \mathbb{R}$ is a progressively measurable map that satisfies $|\gamma(i, \mathbb{S})-\gamma(i-1, \mathbb{S})| \leq M$ for all $i, \mathbb{S}$. The portfolio value for any $\mathbb{S} \in \Omega_{n}$ is

$$
\tilde{Y}_{N}^{\tilde{\pi}}(\mathbb{S})=x+\sum_{i=0}^{N-1} \gamma(i, \mathbb{S})\left(\mathbb{S}_{i+1}-\mathbb{S}_{i}\right)-\kappa \sum_{i=0}^{N-1} \mathbb{S}_{i}|\gamma(i, \mathbb{S})-\gamma(i-1, \mathbb{S})|,
$$

where as before, we set $\gamma(-1, \mathbb{S}) \equiv 0$.

Consider a European option with payoff $\hat{X}=F(\mathbb{S})$, where $F: \Omega_{n} \rightarrow \mathbb{R}$. We do not make any assumptions on the function $F$. The superreplication price is defined by

$$
\tilde{V}(F)=\inf \left\{x \mid \exists \tilde{\pi}=(x, \gamma) \text { such that } \tilde{Y}_{N}^{\tilde{\pi}}(\mathbb{S}) \geq F(\mathbb{S}) \forall \mathbb{S} \in \Omega_{n}\right\}
$$

Theorem 4.1 For any $F: \Omega_{n} \rightarrow \mathbb{R}$,

$$
\tilde{V}(F)=\sup _{\mathbb{Q} \in \tilde{\mathcal{Q}}_{n}} \mathbb{E}_{\mathbb{Q}}\left[F(\mathbb{S})-M \sum_{k=0}^{N-1}\left(\left|\mathbb{E}_{\mathbb{Q}}\left[\mathbb{S}_{N} \mid \mathcal{F}_{k}\right]-\mathbb{S}_{k}\right|-\kappa \mathbb{S}_{k}\right)^{+}\right],
$$

where $\tilde{\mathcal{Q}}_{n}$ is the set of all probability measures on $\Omega_{n}$ that are supported on a finite set.

Proof We start by establishing the inequality

$$
\tilde{V}(F) \leq \sup _{\mathbb{Q} \in \tilde{\mathcal{Q}}_{n}} \mathbb{E}_{\mathbb{Q}}\left[F(\mathbb{S})-M \sum_{k=0}^{N-1}\left(\left|\mathbb{E}_{\mathbb{Q}}\left[\mathbb{S}_{N} \mid \mathcal{F}_{k}\right]-\mathbb{S}_{k}\right|-\kappa \mathbb{S}_{k}\right)^{+}\right]
$$

In fact, in Lemma 3.5 we used only the above inequality. Without loss of generality, we assume that the right-hand side of (4.1) is finite.

For a positive integer $J \in \mathbb{N}$, consider the finite set $\Omega_{n}^{(J)}:=\{0, h, 2 h, \ldots, J h\}^{N}$ with $h=1 / n$ as before. Define the minimal superreplication cost

$$
\tilde{V}^{J}(F)=\inf \left\{x \mid \exists \tilde{\pi}=(x, \gamma) \text { such that } \tilde{Y}_{N}^{\tilde{\pi}}(\mathbb{S}) \geq F(\mathbb{S}) \forall \mathbb{S} \in \Omega_{n}^{J}\right\} .
$$

The cost $\tilde{V}^{J}(F)$ is in fact equal to the minimal superreplication cost in the multinomial model that is supported on the set $\Omega_{n}^{J}$. Thus, we are in a position to apply Theorem 3.1 in [10] with the penalty function

$$
g(\tilde{s}, v)= \begin{cases}\kappa \tilde{s}|\nu| & \text { if }|\nu| \leq M \\ +\infty & \text { otherwise }\end{cases}
$$

The function $g$ is convex in the second variable. Moreover, the convex dual of $g$ is given by

$$
\hat{G}(\tilde{s}, y)=\sup _{v \in \mathbb{R}}(v y-g(\tilde{s}, v))=M(|y|-\kappa s)^{+} .
$$


Therefore, Theorem 3.1 in [10] implies that

$$
\begin{aligned}
\tilde{V}^{J}(F) & =\sup _{\mathbb{Q} \in \mathcal{Q}_{n}^{J}} \mathbb{E}_{\mathbb{Q}}\left[F(\mathbb{S})-M \sum_{k=0}^{N-1}\left(\left|\mathbb{E}_{\mathbb{Q}}\left[\mathbb{S}_{N} \mid \mathcal{F}_{k}\right]-\mathbb{S}_{k}\right|-\kappa \mathbb{S}_{k}\right)^{+}\right] \\
& \leq \sup _{\mathbb{Q} \in \tilde{\mathcal{Q}}_{n}} \mathbb{E}_{\mathbb{Q}}\left[F(\mathbb{S})-M \sum_{k=0}^{N-1}\left(\left|\mathbb{E}_{\mathbb{Q}}\left[\mathbb{S}_{N} \mid \mathcal{F}_{k}\right]-\mathbb{S}_{k}\right|-\kappa \mathbb{S}_{k}\right)^{+}\right],
\end{aligned}
$$

where $\mathcal{Q}_{n}^{J}$ is the set of all probability measures on $\Omega_{n}^{J}$.

For every $J \in \mathbb{N}$, there exists a superreplicating portfolio $\tilde{\pi}_{J}=\left(\tilde{V}^{J}(F)+1 / J, \gamma_{J}\right)$ for the multinomial model supported on $\Omega_{n}^{J}$. Namely, take a progressively measurable map $\gamma_{J}:\{0,1, \ldots, N-1\} \times \Omega_{n} \rightarrow \mathbb{R}$ such that $\left|\gamma_{J}(i, \mathbb{S})-\gamma_{J}(i-1, \mathbb{S})\right| \leq M$ for any $i, \mathbb{S}$ and $\tilde{Y}_{N}^{\tilde{\pi}_{J}}(\mathbb{S}) \geq F(\mathbb{S})$ for every $\mathbb{S} \in \Omega_{n}^{J}$. By using a standard a diagonal procedure we construct a subsequence $\left\{\gamma_{J_{i}}\right\}_{i=1}^{\infty}$ such that for any $j=0,1, \ldots, N-1$ and $\mathbb{S} \in \Omega_{n}, \quad \lim _{i \rightarrow \infty} \gamma_{J_{i}}(j, \mathbb{S})$ exists. We denote this limit by $\gamma(j, \mathbb{S})$. Let $x=\liminf _{i \rightarrow \infty} \tilde{V}^{J_{i}}(F)$. Then clearly $\gamma\{0,1, \ldots, N-1\} \times \Omega_{n} \rightarrow \mathbb{R}$ is a progressively measurable map and the portfolio which is given by $\tilde{\pi}=(x, \gamma)$ satisfies $|\gamma(i, \mathbb{S})-\gamma(i-1, \mathbb{S})| \leq M$ for any $i, \mathbb{S}$. Moreover, $\tilde{Y}_{N}^{\tilde{\pi}}(\mathbb{S}) \geq F(\mathbb{S})$ for every $\mathbb{S} \in \Omega_{n}$. This together with (4.2) yields that

$$
\tilde{V}(F) \leq x \leq \sup _{\mathbb{Q} \in \tilde{\mathcal{Q}}_{n}} \mathbb{E}_{\mathbb{Q}}\left[F(\mathbb{S})-M \sum_{k=0}^{N-1}\left(\left|\mathbb{E}_{\mathbb{Q}}\left[\mathbb{S}_{N} \mid \mathcal{F}_{k}\right]-\mathbb{S}_{k}\right|-\kappa \mathbb{S}_{k}\right)^{+}\right]
$$

and (4.1) follows. ity

Finally, by using arguments similar to those on p. 10 in [10], we prove the inequal-

$$
\tilde{V}(F) \geq \sup _{\mathbb{Q} \in \tilde{\mathcal{Q}}_{n}} \mathbb{E}_{\mathbb{Q}}\left[F(\mathbb{S})-M \sum_{k=0}^{N-1}\left(\left|\mathbb{E}_{\mathbb{Q}}\left[\mathbb{S}_{N} \mid \mathcal{F}_{k}\right]-\mathbb{S}_{k}\right|-\kappa \mathbb{S}_{k}\right)^{+}\right]
$$

and complete the proof.

Acknowledgements Research of Dolinsky is partly supported by a career integration grant, CIG618235 and research of Soner is supported by the European Research Council under the grant 228053FiRM, by the ETH Foundation, and by the Swiss Finance Institute. The authors would like to thank Lev Buhovsky, Jan Obłój, and Josef Teichmann for insightful discussions and comments.

\section{Appendix}

In this appendix, we prove Theorem 3.2. We proceed in several lemmas. We first use Lemma 3.1 to reduce the problem to bounded claims. Then, using a compactness argument as in [3], we further reduce it to bounded and continuous claims.

Lemma 5.1 Suppose that (2.8) holds for all bounded upper semicontinuous functions. Then it also holds for all $G$ satisfying Assumption 2.1. 
Proof Suppose that $G$ satisfies Assumption 2.1. Let $\varphi$ be any smooth function satisfying

$$
0 \leq \varphi \leq 1, \quad \varphi(\mathbb{S})=1 \quad \forall\|\mathbb{S}\| \leq 1, \quad \varphi(\mathbb{S})=0 \quad \forall\|\mathbb{S}\| \geq 2 .
$$

For a constant $M>1$, set

$$
\varphi_{M}(\mathbb{S}):=\varphi(\mathbb{S} / M), \quad G_{M}:=G \varphi_{M}
$$

$G_{M}$ is bounded and upper semicontinuous. Then, by the hypothesis, inequality (2.8) and the duality formula stated in Theorem 2.6 hold for $G_{M}$. In view of Assumption 2.1,

$$
\left|G(\mathbb{S})-G_{M}(\mathbb{S})\right| \leq L\left(1+\|\mathbb{S}\|^{2}\right) \chi_{\{\|\mathbb{S}\| \geq M\}}
$$

Let $\alpha_{M}$ be as in Lemma 3.1. Then, for all sufficiently large $M$,

$$
\left|G(\mathbb{S})-G_{M}(\mathbb{S})\right| \leq 2 L \alpha_{M}(\mathbb{S}) .
$$

Since $G_{M}$ satisfies (2.8),

$$
V\left(G_{M}\right) \leq \sup _{\mathbb{Q} \in \mathcal{M}_{\kappa, \mathcal{P}}} \mathbb{E}_{\mathbb{Q}}\left[G_{M}(\mathbb{S})\right] \leq \sup _{\mathbb{Q} \in \mathcal{M}_{\kappa, \mathcal{P}}} \mathbb{E}_{\mathbb{Q}}[G(\mathbb{S})]+2 L \sup _{\mathbb{Q} \in \mathcal{M}_{\kappa, \mathcal{P}}} \mathbb{E}_{\mathbb{Q}}\left[\alpha_{M}(\mathbb{S})\right]
$$

By the subadditivity of the minimal superreplication cost $V$,

$$
V(G) \leq V\left(G_{M}\right)+2 L V\left(\alpha_{M}\right)
$$

Combining the above inequalities and Lemma 3.1, we arrive at

$$
\begin{aligned}
V(G) & \leq \liminf _{M \rightarrow \infty}\left(V\left(G_{M}\right)+2 L V\left(\alpha_{M}\right)\right) \\
& \leq \sup _{\mathbb{Q} \in \mathcal{M}_{\kappa, \mathcal{P}}} \mathbb{E}_{\mathbb{Q}}[G(\mathbb{S})]+2 L \liminf _{M \rightarrow \infty}\left(V\left(\alpha_{M}\right)+\sup _{\mathbb{Q} \in \mathcal{M}_{\kappa, \mathcal{P}}} \mathbb{E}_{\mathbb{Q}}\left[\alpha_{M}(\mathbb{S})\right]\right) \\
& \leq \sup _{\mathbb{Q} \in \mathcal{M}_{\kappa, \mathcal{P}}} \mathbb{E}_{\mathbb{Q}}[G(\mathbb{S})] .
\end{aligned}
$$

The above proof also yields the following equivalence.

Lemma 5.2 Suppose that (2.8) holds for all nonnegative, bounded, uniformly continuous functions. Then it also holds for all $G$ that are bounded and continuous.

Proof Let $G$ be a bounded continuous function. By adding to $G$ an appropriate constant, we may assume that it is nonnegative as well. Given an integer $N$, define $G_{N}$ as before. Since $G_{N}$ is compactly supported and continuous, it is also uniformly continuous. We then proceed exactly as in the previous lemma to conclude the proof.

We need the following elementary result. 
Lemma 5.3 Let $G$ be bounded and upper semicontinuous. Then there exists a uniformly bounded sequence of continuous functions $G_{n}: \mathbb{R}_{+}^{d} \rightarrow \mathbb{R}$ with $G_{n} \geq G$ and

$$
\limsup _{n \rightarrow \infty} G_{n}\left(x_{n}\right) \leq G(x)
$$

for every $x \in \mathbb{R}_{+}^{d}$ and every sequence $\left\{x_{n}\right\}_{n=1}^{\infty} \subset \mathbb{R}_{+}^{d}$ with $\lim _{n \rightarrow \infty} x_{n}=x$.

Proof For $n \in \mathbb{N}$, consider the grid $O_{n}=\left\{\left(\frac{k_{1}}{n}, \ldots, \frac{k_{d}}{n}\right), k_{1}, \ldots, k_{d} \in \mathbb{Z}_{+}\right\}$. Define the function $G_{n}: O_{n} \rightarrow \mathbb{R}_{+}$by

$$
G_{n}(x)=\sup _{u \in \mathbb{R}_{+}^{d},\|u-x\| \leq \frac{2}{n}} G(u), \quad x \in O_{n} .
$$

Next, we extend $G_{n}$ to the domain $\mathbb{R}_{+}^{d}$.

For any $k_{1}, \ldots, k_{d} \in \mathbb{Z}_{+}$and a permutation $\sigma:\{1, \ldots, d\} \rightarrow\{1, \ldots, d\}$, consider the $d$-simplex

$$
U_{k_{1}, \ldots, k_{d}}^{\sigma}=\left\{\left(x_{1}, \ldots, x_{d}\right) \mid \frac{k_{i}}{n} \leq x_{i} \leq \frac{k_{i}+1}{n} \text { and } x_{\sigma(i)} \leq x_{\sigma(j)}, 1 \leq i<j \leq d\right\} .
$$

Fix a simplex $U_{k_{1}, \ldots, k_{d}}^{\sigma}$. Any $u \in U_{k_{1}, \ldots, k_{d}}^{\sigma}$ can be represented uniquely as a convex combination of the simplex vertices $u_{1}, \ldots, u_{d+1}$ (which belong to $O_{n}$ ). Thus, define the continuous function $G_{k_{1}, \ldots, k_{d}}^{n, \sigma}: U_{k_{1}, \ldots, k_{d}}^{\sigma} \rightarrow \mathbb{R}$ by $G_{k_{1}, \ldots, k_{d}}^{n, \sigma}(u)=\sum_{i=1}^{d+1} \lambda_{i} G_{n}\left(u_{i}\right)$, where $\lambda_{1}, \ldots, \lambda_{d+1} \in[0,1]$ with $\sum_{i=1}^{d+1} \lambda_{i}=1$, and $\sum_{i=1}^{d+1} \lambda_{i} u_{i}=u$ are uniquely determined.

Any element $u \in \mathbb{R}_{+}^{d}$ belongs to at least one simplex of the above form. Moreover, observe that if $u$ belongs to two simplexes, say $U_{k_{1}, \ldots, k_{d}}^{\sigma}$ and $U_{k_{1}^{\prime}, \ldots, k_{d}^{\prime}}^{\sigma^{\prime}}$, then $G_{k_{1}, \ldots, k_{d}}^{n, \sigma}(u)=G_{k_{1}^{\prime}, \ldots, k_{d}^{\prime}}^{n, \sigma^{\prime}}(u)$. Thus, we can extend the function $G_{n}: O_{n} \rightarrow \mathbb{R}$ to a function $G_{n}: \mathbb{R}_{+}^{d} \rightarrow \mathbb{R}$ by setting $G_{n}(u)=G_{k_{1}, \ldots, k_{d}}^{n, \sigma}(u)$ for $u \in U_{k_{1}, \ldots, k_{d}}^{\sigma}$, where $k_{1}, \ldots, k_{d} \in \mathbb{Z}_{+}$, and $\sigma:\{1, \ldots, d\} \rightarrow\{1, \ldots, d\}$ is a permutation.

This sequence has the desired properties.

The following result completes the proof of Theorem 3.2.

Lemma 5.4 Suppose that (2.8) holds for all bounded continuous functions. Then it also holds for all bounded upper semicontinuous $G$.

Proof Let $G$ be bounded and upper semicontinuous. Let $G_{n}$ be the sequence of bounded continuous functions constructed in Lemma 5.3. Hence, (2.8) and Theorem 2.6 hold for $G_{n}$.

Using Theorem 2.6, we choose a sequence of probability measures $\mathbb{Q}_{n} \in \mathcal{M}_{\kappa, \mathcal{P}}$ satisfying

$$
\mathbb{E}^{(n)}\left[G_{n}(\mathbb{S})\right]>V\left(G_{n}\right)-\frac{1}{n}
$$


Using similar compactness arguments as in Lemma 3.6, we construct a subsequence $\mathbb{Q}_{n_{\ell}}, \ell \in \mathbb{N}$, that converges weakly to a probability measure $\tilde{\mathbb{Q}} \in \mathcal{M}_{\kappa}, \mathcal{P}$. Recall that the $G_{n}$ are uniformly bounded. Thus, by (5.1) and the Skorokhod representation theorem,

$$
\limsup _{\ell \rightarrow \infty} \mathbb{E}^{\left(n_{\ell}\right)}\left[G_{n_{\ell}}(\mathbb{S})\right] \leq \tilde{\mathbb{E}}[G(\mathbb{S})] .
$$

This together with (5.2) yields that

$$
V(G) \leq \liminf _{n \rightarrow \infty} V\left(G_{n}\right) \leq \liminf _{n \rightarrow \infty} \mathbb{E}^{(n)}\left[G_{n}(\mathbb{S})\right] \leq \tilde{\mathbb{E}}[G(\mathbb{S})] \leq \sup _{\mathbb{Q} \in \mathcal{M}_{\kappa, \mathcal{P}}} \mathbb{E}_{\mathbb{Q}}[G(\mathbb{S})]
$$

This completes the proof.

\section{References}

1. Acciaio, B., Beiglböck, M., Penkner, F., Schachermayer, W., Temme, J.: A trajectorial interpretation of Doob's martingale inequalities. Ann. Appl. Probab. 23, 1494-1505 (2013)

2. Acciaio, B., Beiglböck, M., Schachermayer, W.: Model-free versions of the fundamental theorem of asset pricing and the superreplication theorem. Math. Financ. doi:10.1111/mafi.12060, to appear

3. Beiglböck, M., Henry-Labordère, P., Penkner, F.: Model-independent bounds for option prices: a mass transport approach. Finance Stoch. 17, 477-501 (2013)

4. Brown, H., Hobson, D., Rogers, L.C.G.: Robust hedging of barrier options. Math. Finance 11, 285314 (2001)

5. Bouchard, B., Nutz, M.: Arbitrage and duality in nondominated discrete-time models. Preprint (2013). arXiv: 1305.6008

6. Cox, A.M.G., Obłój, J.: Robust pricing and hedging of double no-touch options. Finance Stoch. 15, 573-605 (2011)

7. Cox, A.M.G., Obłój, J.: Robust hedging of double touch barrier options. SIAM J. Financ. Math. 2, 141-182 (2011)

8. Carr, P., Lee, R.: Hedging variance options on continuous semimartingales. Finance Stoch. 14, 179207 (2010)

9. Dolinsky, Y., Soner, H.M.: Robust hedging and martingale optimal transport in continuous time. Probab. Theory Relat. Fields. doi:10.1007/s00440-013-0531, to appear

10. Dolinsky, Y., Soner, H.M.: Duality and convergence for binomial markets with friction. Finance Stoch. 17, 447-475 (2013)

11. Galichon, A., Henry-Labordère, P., Touzi, N.: A stochastic control approach to no-arbitrage bounds given marginals, with an application to lookback options. Ann. Appl. Probab. 24, 312-336 (2014)

12. Henry-Labordère, P., Obłój, J., Spoida, P., Touzi, N.: Maximum maximum of martingales given marginals. Preprint (2012). arXiv:1203.6877

13. Hobson, D.: Robust hedging of the lookback option. Finance Stoch. 2, 329-347 (1998)

14. Hobson, D.: The Skorokhod embedding problem and model-independent bounds for option prices. In: Carmona, R.A., et al. (eds.) Paris-Princeton Lectures on Mathematical Finance. Lecture Notes in Mathematics, vol. 2003, pp. 267-318. Springer, Berlin (2011)

15. Kabanov, Y., Safarian, M.: Markets with Transaction Costs. Springer, Berlin (2009)

16. Levental, S., Skorohod, A.V.: On the possibility of hedging options in the presence of transaction costs. Ann. Appl. Probab. 7, 410-443 (1997)

17. Obłój, J.: The Skorokhod embedding problem and its offspring. Probab. Surv. 1, 321-392 (2004)

18. Schachermayer, W.: The fundamental theorem of asset pricing under proportional transaction costs in finite discrete time. Math. Finance 14, 19-48 (2004)

19. Soner, H.M., Shreve, S.E., Cvitanić, J.: There is no nontrivial hedging portfolio for option pricing with transaction costs. Ann. Appl. Probab. 5, 327-355 (1995)

20. Strasser, H.: Mathematical Theory of Statistics. Statistical Experiments and Asymptotic Decision Theory. de Gruyter Studies in Mathematics, vol. 7. de Gruyter, Berlin (1985) 